\title{
Alogliptin Benzoate
}

National Cancer Institute

\section{Source}

National Cancer Institute. Alogliptin Benzoate. NCI Thesaurus. Code C75186.

The benzoate salt form of alogliptin, a selective, orally bioavailable, pyrimidinedionebased inhibitor of dipeptidyl peptidase 4 (DPP-4), with hypoglycemic activity. In addition to its effect on glucose levels, alog liptin may inhibit inflammatory responses by preventing the toll-like receptor 4 (TLR-4)-mediated formation of proinflammatory cytokines. 